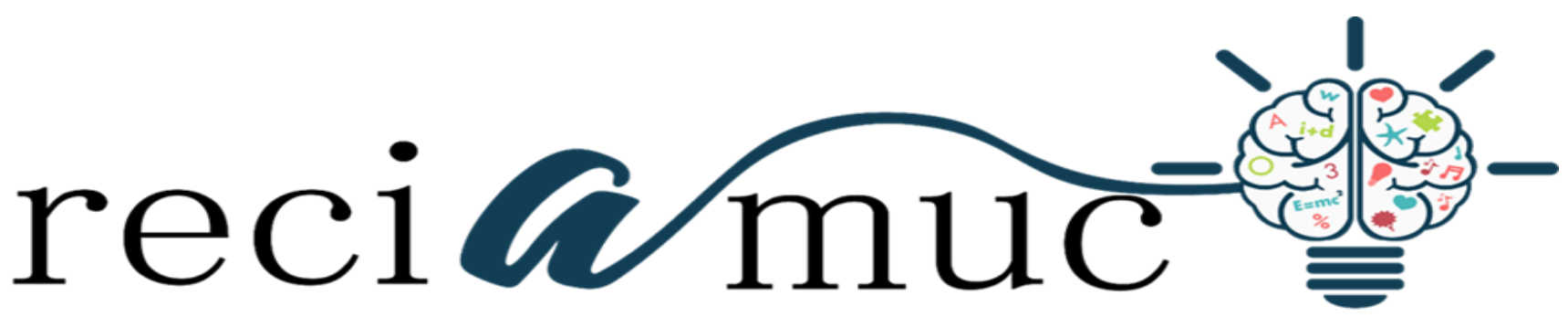

Revista cientifica de investigación actualización del mundo de las ciencias

Lissette Esther Villagrán Rodríguez a; Raúl Enrique Carrión Sánchez b; Aliz Janeth Calero Correa $^{c}$; Dolores Mariela Perero Briones ${ }^{\mathrm{d}}$

Complicaciones más frecuentes en pacientes con pancreatitis

Most frequent complications in patients with pancreatitis

Revista Científica de Investigación actualización del mundo de las Ciencias. Vol. 3 núm., 3, julio, ISSN: 2588-0748, 2018, pp. 838-862

DOI: $10.26820 /$ reciamuc/3.(3).julio.2019.838-862

\section{URL: http://reciamuc.com/index.php/RECIAMUC/article/view/306}

Código UNESCO: 3205 Medicina Interna

Tipo de Investigación: Artículo de Revisión

(C) RECIAMUC; Editorial Saberes del Conocimiento, 2019

Recibido: 28/04/2019

Aceptado: 19/05/2019

Publicado: 01/07/2019

Correspondencia: md.villagran1987@gmail.com

a. Médico; Investigador Independiente; Guayaquil, Ecuador; md.villagran1987@gmail.com

b. Médico; Investigador Independiente; Guayaquil, Ecuador; dr.raulcarrions@ hotmail.com

c. Médico; Investigador Independiente; Guayaquil, Ecuador; alizcalero@ hotmail.com

d. Médico; Investigador Independiente; Guayaquil, Ecuador; marielaperero@ hotmail.com 


\section{Complicaciones más frecuentes en pacientes con pancreatitis}

Vol. 3, núm. 3., (2019)

Lissette Esther Villagrán Rodríguez; Raúl Enrique Carrión Sánchez; Aliz Janeth Calero Correa;

Dolores Mariela Perero Briones

\section{RESUMEN}

La pancreatitis es la enfermedad que afecta al órgano del páncreas y que se le debe prestar atención temprana, ya que, sus complicaciones son bastante letales y puede involucrar otros órganos. El presente trabajo de investigación brinda una recopilación de información importante que expresa, a través, de su desarrollo las complicaciones más comunes asociadas con la pancreatitis, así como también, los tratamientos necesarios empelados en esta afección. En el transcurso de la pancreatitis, los primeros dos o cuatro días de síntomas son los más importantes porque este es el período durante el cual el $15 \%$ al $25 \%$ de los pacientes evolucionan a su forma grave. Según datos clínicos y experimentales, este periodo se caracteriza por un estado inicial de hipovolemia. Se sabe que la morbilidad de la pancreatitis aguda grave aparece en dos etapas. Las primeras dos semanas se caracterizan por un síndrome de respuesta inflamatoria sistémica (SIRS), que resulta de la liberación de mediadores inflamatorios. La insuficiencia orgánica es frecuente y suele ocurrir incluso en ausencia de infección. La tasa de mortalidad temprana es del 42 al 60\%. La segunda etapa comienza aproximadamente dos semanas después del inicio de los síntomas y se caracteriza por complicaciones relacionadas con la sepsis que resultan de la infección de la necrosis pancreática. Por lo tanto, existe una asociación con complicaciones sistémicas, como la insuficiencia pulmonar, la insuficiencia renal y la insuficiencia cardiovascular, conocida como síndrome de insuficiencia múltiple de órganos (MOFS, por sus siglas en inglés).

Palabras Claves: Pancreatitis; Complicaciones; Pancreas; Pseudoquistes. 


\title{
Complicaciones más frecuentes en pacientes con pancreatitis
}

Vol. 3, núm. 3., (2019)

Lissette Esther Villagrán Rodríguez; Raúl Enrique Carrión Sánchez; Aliz Janeth Calero Correa; Dolores Mariela Perero Briones

\begin{abstract}
Pancreatitis is the disease that affects the organ of the pancreas and that should be given early attention, since its complications are quite lethal and may involve other organs. This research work provides a collection of important information that expresses, through its development, the most common complications associated with pancreatitis, as well as the necessary treatments used in this condition. In the course of pancreatitis, the first two or four days of symptoms are the most important because this is the period during which $15 \%$ to $25 \%$ of patients progress to their severe form. According to clinical and experimental data, this period is characterized by an initial state of hypovolemia. It is known that the morbidity of severe acute pancreatitis appears in two stages. The first two weeks are characterized by a systemic inflammatory response syndrome (SIRS), which results from the release of inflammatory mediators. Organ failure is frequent and usually occurs even in the absence of infection. The early mortality rate is 42 to $60 \%$. The second stage begins approximately two weeks after the onset of symptoms and is characterized by complications related to sepsis that result from pancreatic necrosis infection. Therefore, there is an association with systemic complications, such as pulmonary insufficiency, renal failure and cardiovascular insufficiency, known as multiple organ failure syndrome (MOFS, for its acronym in English).
\end{abstract}

Key Words: Pancreatitis; Complications; Pancreas; Pseudocysts 


\section{Complicaciones más frecuentes en pacientes con pancreatitis}

Vol. 3, núm. 3., (2019)

Lissette Esther Villagrán Rodríguez; Raúl Enrique Carrión Sánchez; Aliz Janeth Calero Correa;

Dolores Mariela Perero Briones

\section{Introducción.}

El cuerpo humano está compuesto por una gran cantidad de células que se agrupan en tejidos y que se organizan en órganos, y éstos en ocho aparatos o sistemas: locomotor (muscular y óseo), respiratorio, digestivo, excretor, circulatorio, endocrino, nervioso y reproductor. Los órganos como el hígado, la vesícula biliar, el páncreas y el intestino delgado pertenecen al sistema digestivo y están conectados a través de una red de tubos llamados conductos biliares.

El autor (Medicine, 2018 ) expresa, el páncreas es un órgano alargado y angosto que está ubicado en la parte de atrás del abdomen y detrás del estómago. La parte derecha del órgano, llamada la cabeza, es la más ancha, y se ubica en la curva del duodeno, que es la primera porción del intestino delgado. La parte izquierda, llamada el cuerpo del páncreas, es angosta y se extiende ligeramente hacia arriba y termina en la parte llamada cola, que está cerca del bazo.

La pancreatitis es un proceso inflamatorio agudo del páncreas, que tiene afectación variable de otros tejidos regionales y órganos o remotos. La enfermedad puede ser clasificada como leve o grave dependiendo de la extensión de la inflamación y la afectación de órganos.

El tipo de pancreatitis leve consiste en una pancreatitis intersticial (edematosa) con disfunción de órganos extrapancreático mínima o de ninguna participación, mientras que la pancreatitis grave se manifiesta con la insuficiencia de órganos, además de complicaciones locales tales como pseudoquistes, necrosis, formación de abscesos, fistulización, o complicaciones vasculares. (Tercero, 2013) 


\section{Complicaciones más frecuentes en pacientes con pancreatitis}

Vol. 3, núm. 3., (2019)

Lissette Esther Villagrán Rodríguez; Raúl Enrique Carrión Sánchez; Aliz Janeth Calero Correa; Dolores Mariela Perero Briones

El objetivo central de este trabajo investigativo, es estudiar las complicaciones comunes que se producen en pacientes que padecen de esta enfermedad, así como también, considerar los tratamientos para disminuir la posibilidad de desarrollar pancreatitis grave en aquellas personas que ya presentan un cuadro de pancreatitis leve.

\section{Métodos y materiales.}

Para el desarrollo de este proceso investigativo, se plantea como metodología la encaminada hacia una orientación científica particular que se encuentra determinada por la necesidad de indagar en forma precisa y coherente una situación, en tal sentido (Davila, 2015) define la metodología "como aquellos pasos previos que son seleccionados por el investigador para lograr resultados favorables que le ayuden a plantear nuevas ideas".(p.66)

Lo citado por el autor, lleva a entender que el desarrollo de la acción investigativa busca simplemente coordinar acciones enmarcadas en una revisión bibliográfica con el fin de complementar ideas previas relacionadas Complicaciones más frecuentes en pacientes con pancreatitis, a través de una revisión de literatura, para así finalmente elaborar un cuerpo de consideraciones generales que ayuden a ampliar el interés propuesto.

\section{Tipo de Investigación}

Dentro de toda práctica investigativa, se precisan acciones de carácter metodológico mediante las cuales, se logra conocer y proyectar los eventos posibles que la determinan, así como las características que hacen del acto científico un proceso interactivo ajustado a una 


\section{Complicaciones más frecuentes en pacientes con pancreatitis}

Vol. 3, núm. 3., (2019)

Lissette Esther Villagrán Rodríguez; Raúl Enrique Carrión Sánchez; Aliz Janeth Calero Correa;

Dolores Mariela Perero Briones

realidad posible de ser interpretada. En este sentido, se puede decir, que la presente investigación corresponde al tipo documental, definido por Castro (2016), "se ocupa del estudio de problemas planteados a nivel teórico, la información requerida para abordarlos se encuentra básicamente en materiales impresos, audiovisuales y /o electrónicos”. (p.41).

En consideración a esta definición, la orientación metodológica permitió la oportunidad de cumplir con una serie de actividades inherentes a la revisión y lectura de diversos documentos donde se encontraron ideas explicitas relacionadas con los tópicos encargados de identificar a cada característica insertada en el estudio. Por lo tanto, se realizaron continuas interpretaciones con el claro propósito de revisar aquellas apreciaciones o investigaciones propuestas por diferentes investigadores relacionadas con el tema de interés, para luego dar la respectiva argumentación a los planteamientos, en función a las necesidades encontradas en la indagación.

\section{Fuentes Documentales}

El análisis correspondiente a las características que predomina en el tema seleccionado, llevan a incluir diferentes fuentes documentales encargadas de darle el respectivo apoyo y en ese sentido cumplir con la valoración de los hechos a fin de generar nuevos criterios que sirven de referencia a otros procesos investigativos. Para (CASTRO, 2016) las fuentes documentales incorporadas en la investigación documental o bibliográfica, "representa la suma de materiales sistemáticos que son revisados en forma rigurosa y profunda para llegar a un análisis del fenómeno".(p.41). Por lo tanto, se procedió a cumplir con la realización de una lectura previa determinada para encontrar aquellos aspectos estrechamente vinculados con el tema, con el fin de explicar mediante un desarrollo las respectivas apreciaciones generales de importancia. 


\section{Complicaciones más frecuentes en pacientes con pancreatitis}

Vol. 3, núm. 3., (2019)

Lissette Esther Villagrán Rodríguez; Raúl Enrique Carrión Sánchez; Aliz Janeth Calero Correa; Dolores Mariela Perero Briones

\section{Técnicas para la Recolección de la Información}

La conducción de la investigación para ser realizada en función a las particularidades que determinan a los estudios documentales, tiene como fin el desarrollo de un conjunto de acciones encargadas de llevar a la selección de técnicas estrechamente vinculadas con las características del estudio. En tal sentido, (Bolívar, 2015), refiere, que es "una técnica particular para aportar ayuda a los procedimientos de selección de las ideas primarias y secundarias”. (p. 71).

Por ello, se procedió a la utilización del subrayado, resúmenes, fichaje, como parte básica para la revisión y selección de los documentos que presentan el contenido teórico. Es decir, que mediante la aplicación de estas técnicas se pudo llegar a recoger informaciones en cuanto a la revisión bibliográfica de los diversos elementos encargados de orientar el proceso de investigación. Tal como lo expresa, (Bolívar, 2015) "las técnicas documentales proporcionan las herramientas esenciales y determinantes para responder a los objetivos formulados y llegar a resultados efectivos" (p. 58). Es decir, para responder con eficiencia a las necesidades investigativas, se introdujeron como técnica de recolección el método inductivo, que hizo posible llevar a cabo una valoración de los hechos de forma particular para llegar a la explicación desde una visión general.

Asimismo, se emplearon las técnicas de análisis de información para la realización de la investigación que fue ejecutada bajo la dinámica de aplicar diversos elementos encargados de determinar el camino a recorrer por el estudio, según, (Bolívar, 2015) las técnicas de procesamiento de datos en los estudios documentales "son las encargadas de ofrecer al investigador la visión o pasos que debe cumplir durante su ejercicio, cada una de ellas debe estar 


\section{Complicaciones más frecuentes en pacientes con pancreatitis}

Vol. 3, núm. 3., (2019)

Lissette Esther Villagrán Rodríguez; Raúl Enrique Carrión Sánchez; Aliz Janeth Calero Correa; Dolores Mariela Perero Briones

en correspondencia con el nivel a emplear" (p. 123). Esto indica, que para llevar a cabo el procesamiento de los datos obtenidos una vez aplicado las técnicas seleccionadas, tales como: fichas de resumen, textual, registros descriptivos entre otros, los mismos se deben ajustar al nivel que ha sido seleccionado.

\section{Resultados.}

La pancreatitis presenta complicaciones una vez se convierte en grave, las más comunes son pseudoquistes, necrosis, formación de abscesos, fistulización, o complicaciones vasculares, además de presentar insuficiencia de algunos órganos.

\section{Pseudoquistes}

Los Seudoquistes se pueden desarrollar después de un episodio de la pancreatitis aguda en aproximadamente un 10\% de los casos. (Cameron, 2014). Un seudoquiste pancreático es una colección circunscrita, maduración de las secreciones pancreáticas encajonadas en el tejido de granulación que ocurre en o alrededor del páncreas como resultado de la pancreatitis inflamatoria con o sin interrupción ductal como se observa en la Figura 1. Los seudoquistes representan aproximadamente el $80 \%$ de las lesiones quísticas del páncreas. Ellos pueden ser únicos o múltiples, dentro o fuera del páncreas y puede variar en tamaño. 


\section{Complicaciones más frecuentes en pacientes con pancreatitis}

Vol. 3, núm. 3., (2019)

Lissette Esther Villagrán Rodríguez; Raúl Enrique Carrión Sánchez; Aliz Janeth Calero Correa; Dolores Mariela Perero Briones

\section{Figura $N^{o} 1$. Seudoquiste pancreático}

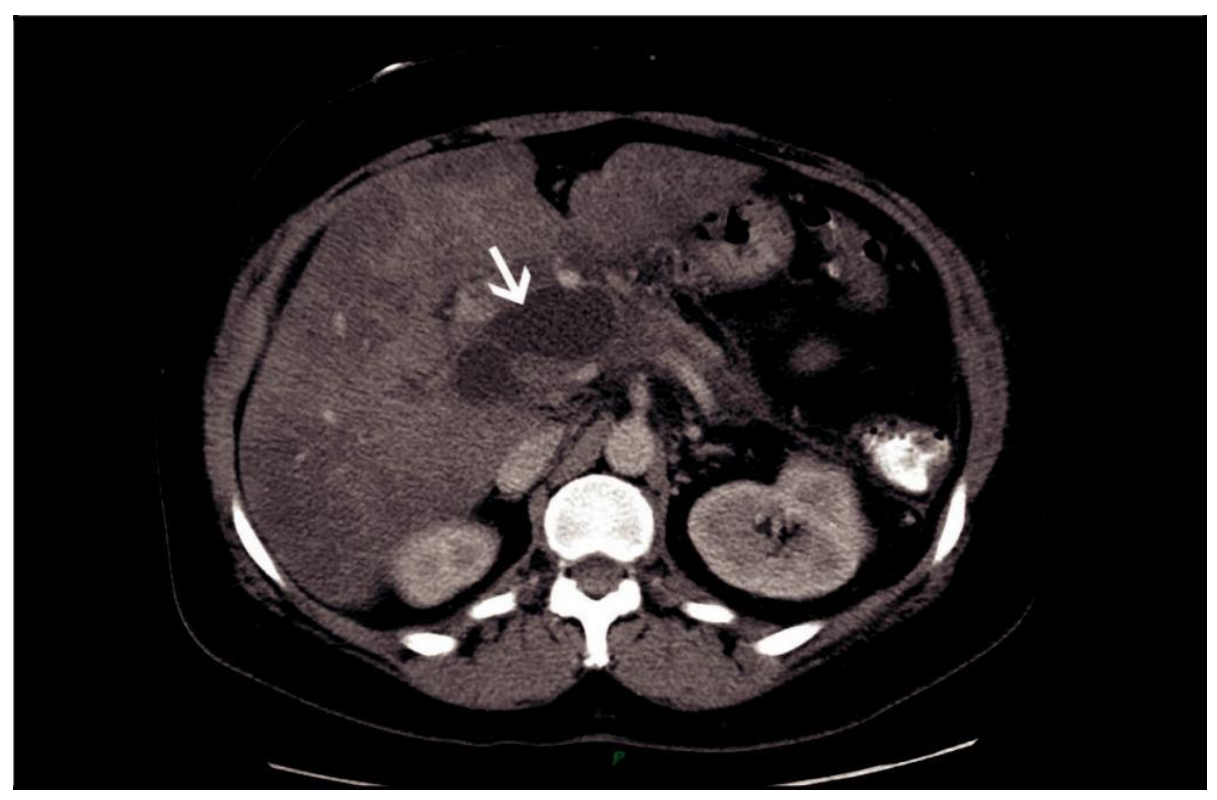

Fuente: (Cameron, 2014).

La mayoría de los pseudoquistes comunican con el sistema ductal pancreático y contienen altas concentraciones de enzimas digestivas. Las paredes de un seudoquiste están formadas por las estructuras adyacentes, que pueden incluir el estómago, colon transverso, epiplón gastrocólico, y el propio páncreas. La característica clave de un seudoquiste es el revestimiento, que contiene material fibroso y tejido de granulación y no tiene verdadera revestimiento epitelial. Esto distingue a los pseudoquistes de una verdadera lesión quística del páncreas. (Brooks, 2013)

Ha habido dos hipótesis sugeridas para la formación de pseudoquistes como resultado de la pancreatitis aguda. La primera es que la inflamación pancreática puede resultar en necrosis de tejido pancreático y peripancreática, que puede entonces progresar en la licuefacción del parénquima y el tejido circundante con la organización subsiguiente y eventual evolución en un 


\section{Complicaciones más frecuentes en pacientes con pancreatitis}

Vol. 3, núm. 3., (2019)

Lissette Esther Villagrán Rodríguez; Raúl Enrique Carrión Sánchez; Aliz Janeth Calero Correa;

Dolores Mariela Perero Briones

seudoquiste. La segunda hipótesis sugiere que la necrosis del parénquima pancreático ductal conduce a la interrupción y la fuga bruto de líquido pancreático, que posteriormente se somete a la organización y la evolución en un seudoquiste.

La clasificación de estas colecciones de líquido pancreáticas como pseudoquistes frente a la necrosis pancreática, abscesos pancreáticos, o colecciones de líquido peripancreáticos puede ser difícil. La nomenclatura de los seudoquistes pancreáticos se acordó por consenso de los expertos en el Simposio de Atlanta que define un seudoquiste como una colección de líquido que fue $>4$ semanas de edad y rodeado por una pared bien definida; Sin embargo, en la práctica clínica, colecciones de líquido encontraron en las imágenes durante y después de un episodio de pancreatitis aguda no puede ser clasificado tan fácilmente. (Baron TH, 2002)

Si el diagnóstico es cuestionable, la prueba adicional puede llevar a cabo, incluida la aspiración de líquido debajo de la tomografía computarizada (TC) o una ecografía endoscópica con orientación (USE). En comparación con los otros tipos de colecciones de líquido pancreáticas agudas, los seudoquistes pancreáticos tienden a tener mayores concentraciones de enzimas pancreáticas y un mínimo o ningún resto de tejido y son en su mayor parte estéril. El diagnóstico de pseudoquiste también puede ser apoyado por la presencia de ascitis pancreática o derrame pleural pancreático que tienen altas concentraciones de amilasa> 1000 UI / L.7. (Baron TH, 2002)

En un estudio realizado en un centro de alcoholicos ubicado en la ciudad de Lima y realizado por (Bradley L, 2011) a personas que presentaban una alta incidencia de pancreatitis alcohólica aguda, se aplicó tratamiento tradicional de estudio clásico que sugiere que un 


\section{Complicaciones más frecuentes en pacientes con pancreatitis}

Vol. 3, núm. 3., (2019)

Lissette Esther Villagrán Rodríguez; Raúl Enrique Carrión Sánchez; Aliz Janeth Calero Correa; Dolores Mariela Perero Briones

seudoquiste que persiste durante más de 6 semanas rara vez se resuelven y tiene una tasa de complicaciones representando casi el 50\% durante la observación continua. Las observaciones de este estudio no se pueden tomar como referencia en otras poblaciones, debido a la condición de alcoholismo de la población estudiada. Por tal razón datos recientes sugieren que un seudoquiste asintomática no requiere tratamiento independientemente de su tamaño. (Yeo J, 2010)

En otro estudio realizado por (Vitas GJ, 2002) a 68 pacientes con vidas conservadoras que presentaron seudoquiste, mostró que sólo había una incidencia del 9\% de complicaciones mayores incluyendo perforación, pseudoaneurisma, y formación de abscesos, 27\% de los pacientes fueron sometidos a cirugía electiva debido a la ampliación del seudoquiste con el dolor asociado, 63\% de los pacientes se mantuvieron bien sin síntomas o complicaciones durante una media de 51 meses. Estos hallazgos sugieren que puede ser satisfactorio para controlar seudoquistes asintomáticos con imágenes en serie.

El síntoma más frecuente de un seudoquiste es dolor abdominal superior, pero otros síntomas menos comunes incluyen saciedad temprana, náuseas, vómitos, ictericia, prurito, edema, o sangrado gastrointestinal. Estos síntomas se correlacionan a la obstrucción de las estructuras locales tales como la salida gástrica, IVC, conducto biliar común, intestino delgado, colon, bazo, o venas porta y por lo general están relacionadas con el tamaño de pseudoquistes y su ubicación. Ocasionalmente, un seudoquiste puede romperse en el estómago en el intestino delgado ver Figura 2. Otras complicaciones reportadas atribuidas a seudoquistes incluyen infección, hemorragia intraquística, o ruptura, lo que lleva a la ascitis pancreática. Los pseudoquistes también se ha reportado que han migrar al pecho, así como otros lugares poco usuales. (Yeo J, 2010). En el contexto clínico de un seudoquiste relacionada con pancreatitis 


\section{Complicaciones más frecuentes en pacientes con pancreatitis}

Vol. 3, núm. 3., (2019)

Lissette Esther Villagrán Rodríguez; Raúl Enrique Carrión Sánchez; Aliz Janeth Calero Correa;

Dolores Mariela Perero Briones

aguda, nuevos síntomas de dolor abdominal, fiebre, o escalofríos debe ser reconocido como posible aparición de una infección o formación de abscesos, y apropiado quirúrgica, endoscópica, o la gestión radiológico debe llevarse a cabo en función de la presentando síntomas y hallazgos.

Figura $N^{o}$ 2. Pseudoquiste ruptura en el duodeno visto en la endoscopia superior. (B) La visualización de la cavidad del quiste endoscópicamente

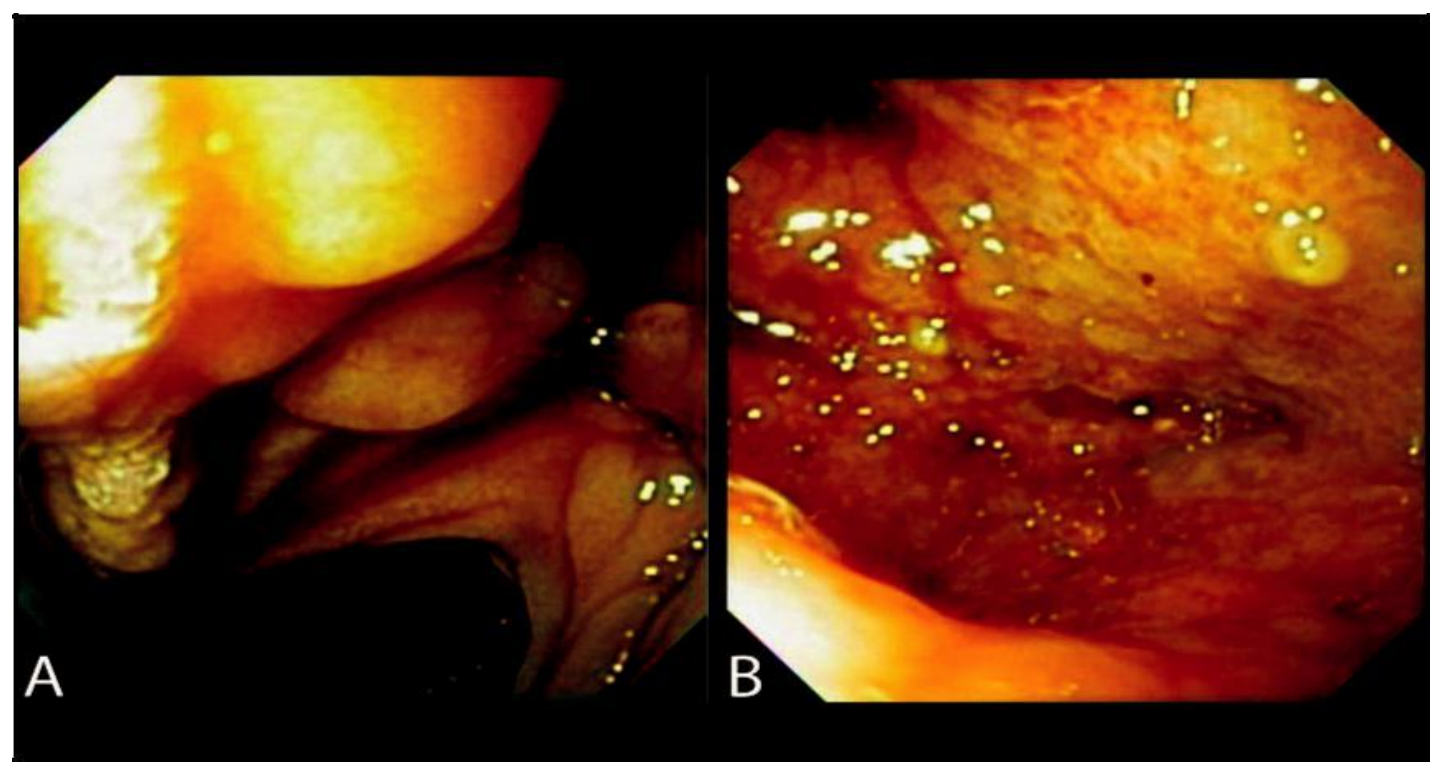

Fuente: (Yeo J, 2010).

\section{Drenaje}

El drenaje quirúrgico fue en un tiempo la única forma de terapia disponible para pseudoquistes sintomáticos o pseudoquistes que requirian drenaje debido a la infección. Sin embargo, durante los últimos 10 años, el drenaje percutáneo guiado por imágenes y el drenaje endoscópico se han vuelto cada vez más populares. Hasta la fecha, no existen estudios 


\section{Complicaciones más frecuentes en pacientes con pancreatitis}

Vol. 3, núm. 3., (2019)

Lissette Esther Villagrán Rodríguez; Raúl Enrique Carrión Sánchez; Aliz Janeth Calero Correa; Dolores Mariela Perero Briones

aleatorizados comparativos que evalúan la eficacia o la morbilidad asociada a estas modalidades de drenaje. La experiencia local ha impulsado el uso de un método sobre otro en la determinación de cuál es el enfoque más adecuado. (Catalano M, 2015)

El drenaje quirúrgico sigue siendo la modalidad más aceptado para la gestión de seudoquistes pancreáticos. La literatura quirúrgica describe métodos laparoscópicos más nuevos para el drenaje, aunque la mayoría de los datos provienen de los procedimientos de drenaje quirúrgicos abiertos. El drenaje quirúrgico abierto puede llevarse a cabo a través de cistgastrostomia, cistenterontomia (con o sin creación de una rama de Roux), o la resección quirúrgica. Descrita por primera vez en 1994, se han utilizado varios enfoques diferentes de drenaje laparoscópico, cistgastrostomia y laparoscópica se puede realizar utilizando un enfoque transgástrica anterior que requiere una gastrostomía anterior para el acceso a la creación cistgastrostomia a través de la pared gástrica posterior. (Parque E, 2002)

Un enfoque posterior también se ha descrito con creación de un único gastrostomía en continuidad con el pseudoquiste a través del saco menor. Este último es considerado técnicamente más fácil y se asocia con menor sangrado intraoperatorio. (Cervantes A, 2004)

En general, la morbilidad y la mortalidad asociadas con drenaje quirúrgico se ha demostrado que es sustancial con tasas del $25 \%$ y $5 \%$, respectivamente se ha informado de algunas series de tasas de recurrencia seudoquiste después de drenaje quirúrgico para ser tan alta $15 \%$, y pueden ser más frecuentes si el conducto pancreático principal se obstruye aguas abajo de la anastomosis quirúrgica. El colangiopancreatografía preoperatorio retrógrado endoscópico (CPRE) es por lo general realizado para determinar si existe una obstrucción ductal, y en tales 


\section{Complicaciones más frecuentes en pacientes con pancreatitis}

Vol. 3, núm. 3., (2019)

Lissette Esther Villagrán Rodríguez; Raúl Enrique Carrión Sánchez; Aliz Janeth Calero Correa; Dolores Mariela Perero Briones

casos, las resecciones seudoquiste se puede ofrecer con drenaje de catéter percutáneo de imagen guiada que ha informado que es tan eficaz como el tratamiento quirúrgico de un seudoquiste si el quiste es estéril o infectada. (Adams DB, 2015) Sin embargo, hay varias complicaciones asociadas con este procedimiento, con las infecciones más comunes de drenaje-pista que está siendo, que se produce en hasta el $50 \%$ de los casos.

La complicación más preocupante es la formación de una fístula externa. Este riesgo está asociado con ninguna intervención catéter percutáneo, pero en el contexto de drenaje seudoquiste con alta concentración de enzimas pancreáticas, es mucho más alto, la anatomía ductal se ha demostrado que se correlaciona tanto con el éxito del método percutáneo, así como el desarrollo de complicaciones después del drenaje percutáneo. Los eudoquistes con conductos pancreáticos normales y aquellos asociados con estenosis del conducto pancreático sin disrupción del conducto o comunicación quiste tienen mayores tasas de drenaje éxito. Las estenosis en asociación con una interrupción del conducto pancreático y la comunicación quiste y los asociados con completa de corte del conducto pancreático tienen resultados relativamente pobres. Las últimas características también predisponen a largo plazo la formación de fístulas externa de páncreas. CPRE se lleva a cabo por lo general para determinar la anatomía ductal antes del drenaje percutáneo. Las anatomías mal definidas se considera que es una contraindicación relativa para el drenaje percutáneo de imagen guiada.

La descompresión pseudoquiste se puede lograr mediante dos métodos endoscópicos, el drenaje transpapiario, a través, de la colocación de stents, es decir, de la ampolla directamente en el propio seudoquiste o mediante la creación de un cistgastrostomia o cistduodenostomía endoscópicamente colocado. (Lawson M, 2011). Los estudios no han demostrado una diferencia 


\section{Complicaciones más frecuentes en pacientes con pancreatitis}

Vol. 3, núm. 3., (2019)

Lissette Esther Villagrán Rodríguez; Raúl Enrique Carrión Sánchez; Aliz Janeth Calero Correa; Dolores Mariela Perero Briones

significativa en los resultados de los enfoques respectivos en el drenaje del pseudoquiste. Independientemente de la técnica utilizada, se retira el catéter después de tres a cuatro semanas si el cierre del seudoquiste se ve en la imagen. La resolución a largo plazo con drenaje endoscópico exitoso de seudoquistes se ha reportado en la mayoría de series, con tasas que van del $65 \%$ al 89\%. La principal complicación con drenaje endoscópico del seudoquiste es la hemorragia, perforación retroperitoneal, y la infección. El sangrado ha sido considerado como el más importante, con alrededor de un 5\% de riesgo de requerir intervenciones quirúrgicas. Algunas series han sugerido que el drenaje nasoquístico con riego puede prevenir la oclusión y puede ser utilizado en seudoquistes infectados o posiblemente incluso en necrosis organizada. Las tasas de recurrencia han sido reportados en un rango de 6\% a 18\%; Sin embargo, las tasas pueden reducirse con stent conducto pancreático a largo plazo en pacientes con disrupción del conducto grave. (Arvanitakis M, 2007)

La pancreatitis necrotizante y la formación de abscesos

La necrosis pancreática es una complicación local que puede ocurrir hasta en el 10\% $20 \%$ de los pacientes que sufren de pancreatitis aguda. El diagnóstico puede hacerse sobre la base de formación de imágenes y se define por la presencia de más del 30\% de no mejora del páncreas en la TC con contraste o resonancia magnética (MRI). La necrosis infectada se refiere a la contaminación bacteriana de tejido pancreático necrótico en ausencia de formación de abscesos. Se puede progresar a la formación de abscesos y se define como una colección de pus resultante de la licuefacción infectada de tejido pancreático necrótico. Los pseudoquistes infectados, como se describió anteriormente, son una colección de líquido infectado con altas 


\section{Complicaciones más frecuentes en pacientes con pancreatitis}

Vol. 3, núm. 3., (2019)

Lissette Esther Villagrán Rodríguez; Raúl Enrique Carrión Sánchez; Aliz Janeth Calero Correa;

Dolores Mariela Perero Briones

concentraciones de enzimas pancreáticas dentro de una pared fibrosa definido que carece de revestimiento epitelial subyacente. (Berger HG, 2003)

Aunque hay una superposición en la caracterización de infecciones localizadas del páncreas, reconociendo la nomenclatura que describe la complicación tiene importancia con respecto a la gestión. La Figura 3 muestra evidencia de pared fuera de la necrosis pancreática en la TC.

\section{Figura $N^{o} 3$. Coronal de imágenes TC que muestra la necrosis pancreática paredes-off}

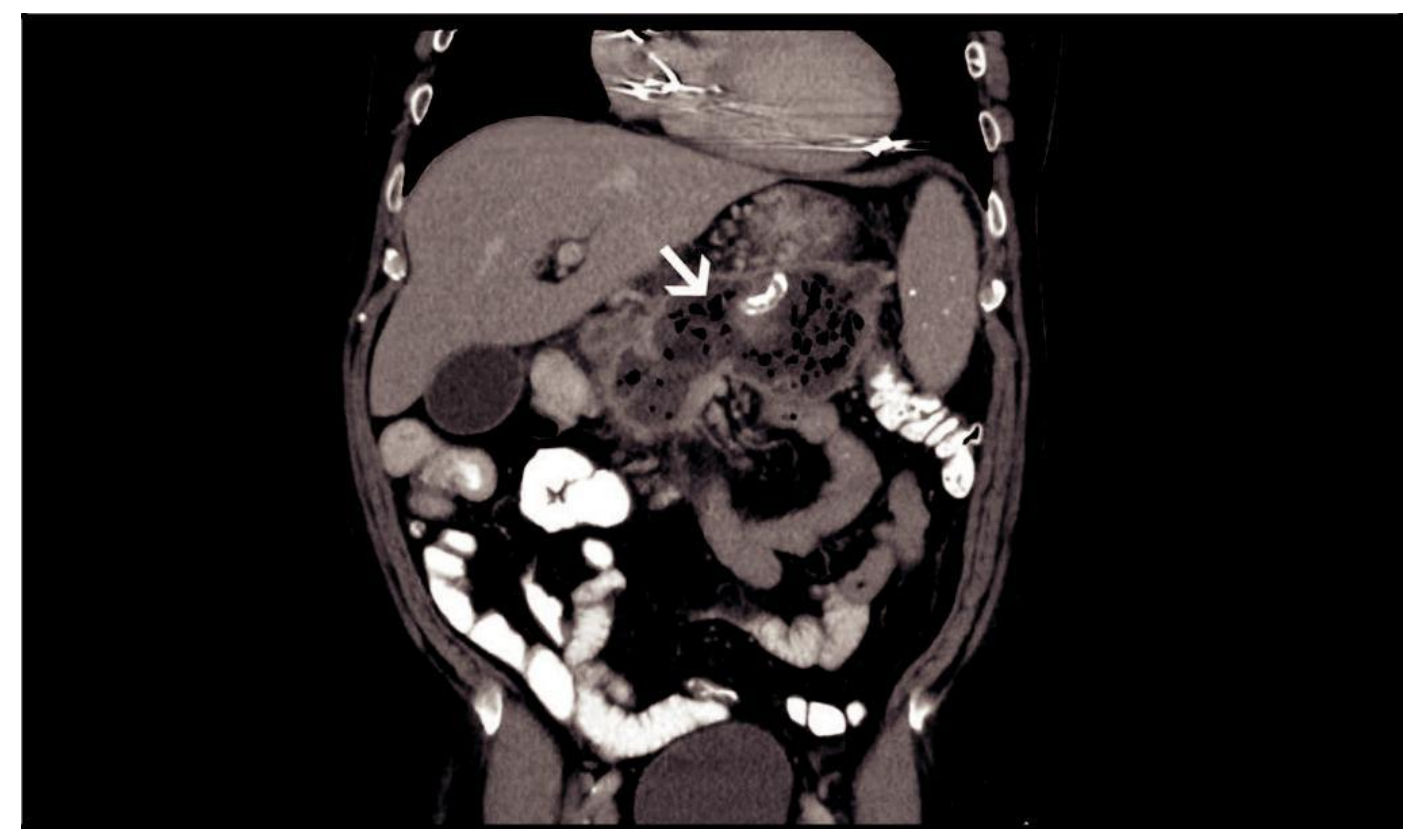

Fuente: (Berger HG, 2003)

La necrosis estéril

La necrosis pancreática estéril se produce temprano en el curso de la pancreatitis y se ve típicamente dentro de los primeros 10 a 14 días de la enfermedad. El contraste CT es el estándar 


\section{Complicaciones más frecuentes en pacientes con pancreatitis}

Vol. 3, núm. 3., (2019)

Lissette Esther Villagrán Rodríguez; Raúl Enrique Carrión Sánchez; Aliz Janeth Calero Correa; Dolores Mariela Perero Briones

de oro en el diagnóstico de la pancreatitis necrotizante. El desarrollo de la necrosis es un proceso continuo que se produce dentro de horas de inicio de los síntomas, sin embargo, el contraste CT mejora en los primeros días se perderá la formación de parénquima necrótico y puede ser engañosa. La sensibilidad de la TC con contraste para la necrosis pancreática se acerca a $100 \%$ entre 4 a 10 días después de la aparición de pancreatitis. (Dervenis C, 2012). Esta es la forma más grave de la inflamación pancreatitica. La cantidad de tejido necrótico es el más fuerte predictor de la mortalidad en pancreatitis necrotizante.

Después del diagnóstico, el tratamiento es el cuidado de apoyo máxima incluyendo la nutrición y la prevención de infecciones de las vías intravenosas. El papel de los antibióticos profilácticos para prevenir la infección se ha demostrado que es cuestionable y no se recomienda. El desbridamiento quirúrgico de la necrosis pancreática estéril no ha demostrado que mejora la morbilidad o la mortalidad en la mayoría de los pacientes. Sin embargo, el desbridamiento quirúrgico es a menudo necesario cuando la necrosis se infecta.

\section{La necrosis infectada}

La necrosis pancreática infectada se produce generalmente después de 10 a 14 días de la enfermedad. La infección bacteriana es un determinante importante de mortalidad, complicaciones sistémicas graves son comunes en pacientes con necrosis estéril, pero las tasas de mortalidad son relativamente bajos, con una incidencia de 5\% 10\%. (Buchler MW, 2000). Con el desarrollo de la infección pancreática, la mortalidad aumenta a 20\% 30\%, a pesar de la desbridamiento quirúrgica. El diagnóstico de la infección se puede realizar mediante aspiración con aguja fina (FNA) guiada por TC, la FNA ha estado a salvo en el diagnóstico de la necrosis 


\section{Complicaciones más frecuentes en pacientes con pancreatitis}

Vol. 3, núm. 3., (2019)

Lissette Esther Villagrán Rodríguez; Raúl Enrique Carrión Sánchez; Aliz Janeth Calero Correa; Dolores Mariela Perero Briones

infectada. La tinción de Gram solo tiene una sensibilidad de casi el 95\%. En el caso de un FNA negativa con una sospecha persistente para la infección, se debe repetir las aspiraciones realizandolas cada 4 a 7 días.

En los pacientes con diagnóstico de necrosis infectada con insuficiencia de órganos sepsis o persistente, el desbridamiento quirúrgico se debe considerar seriamente. Sin embargo, en pacientes estables con necrosis infectada, la atención de apoyo máxima y el uso de antibióticos deben ser proporcionadas. El momento óptimo para el desbridamiento quirúrgico es de al menos 3 a 4 semanas después de la aparición de la enfermedad. El desbridamiento retardado permite la estabilización clínica del paciente, la resolución de la disfunción de órganos, el rebajado en la reacción inflamatoria en el retroperitoneo, y la delimitación de tejidos vivos y muertos a través de la organización y la formación de una pared fibrosa. Esta entidad clínica se conoce como-off de paredes necrosis pancreática (WOPN). Los beneficios de la espera de WOPN son la resolución de la inflamación sistémica y la determinación de la delimitación de tejidos vivos y muertos que daría lugar a métodos menos invasivos de desbridamiento. Sin embargo, desbridamiento de fase temprana dentro de los primeros 3 a 4 semanas requiere un enfoque quirúrgico abierto donde como el desbridamiento de fase tardía de WOPN puede ser tratado laparoscópicamente, percutánea o por vía endoscópica.

\section{Absceso pancreático}

Absceso pancreático es una complicación tardía de la pancreatitis aguda necrotizante, que se produce más de 4 semanas después de la enfermedad inicial. La gestión y complicaciones son similares a la de los pseudoquistes infectados. La formación de un absceso puede causar efectos 


\section{Complicaciones más frecuentes en pacientes con pancreatitis}

Vol. 3, núm. 3., (2019)

Lissette Esther Villagrán Rodríguez; Raúl Enrique Carrión Sánchez; Aliz Janeth Calero Correa; Dolores Mariela Perero Briones

de la presión y la obstrucción por la compresión de las estructuras circundantes, incluyendo el colon, estómago, duodeno, y el conducto biliar común. La tasa de mortalidad asociada con absceso pancreático es generalmente mucho menor que la de necrosis infectada. Se trata inicialmente con antibióticos seguido de un drenaje quirúrgico, percutánea, o endoscópica de ser necesaria. Los abscesos que son drenados por vía percutánea o por vía endoscópica, que no muestran mejoría clínica deben someterse a un drenaje quirúrgico inmediato. (Buchler MW, 2000)

Las complicaciones vasculares

La incidencia de complicaciones vasculares relacionadas con pancreatitis aguda no se conoce, aunque se ha especulado tener una incidencia a punto de $25 \%$ en los casos de pancreatitis aguda. Las complicaciones más frecuentes son la hemorragia pseudoquistes, erosiones de los vasos gastrointestinales, trombosis venosa, la formación de varices, y la formación de pseudoaneurisma. La patogénesis de complicaciones vasculares es multifactorial, pero los mediadores predominantes son la diseminación local de la inflamación, los efectos de irritación de las enzimas pancreáticas activadas, y necrosis por presión debido a la recogida de fluido o restos inflamatorios en las estructuras circundantes. La mortalidad relacionada con la hemorragia asociada con estas complicaciones de la pancreatitis aguda se ha informado a ocurrir hasta en un $14,5 \%$ del tiempo, y no parece ser una correlación con la gravedad de la enfermedad subyacente. (Flati G, 2015) 


\section{Complicaciones más frecuentes en pacientes con pancreatitis}

Vol. 3, núm. 3., (2019)

Lissette Esther Villagrán Rodríguez; Raúl Enrique Carrión Sánchez; Aliz Janeth Calero Correa;

Dolores Mariela Perero Briones

\section{Pseudoaneurisma}

Un pseudoaneurisma es una colección de sangre y coágulos de sangre que se ha formado fuera de un buque. Se han reportado de 3,5\% $10 \%$ de los pseudoaneurismas de pacientes con pancreatitis. Las arterias más comúnmente involucrados son el bazo, gastroduodenal, y pancreaticoduodenal. Estos vasos están involucrados en aproximadamente el $90 \%$ de todos los pseudoaneurismas relacionados con pancreatitis aguda. La rotura de los pseudoaneurismas es una complicación grave de pancreatitis en el sangrado de seudoquiste, el tracto gastrointestinal, cavidad peritoneal o parénquima pancreático. El diagnóstico no siempre está claro como el paciente puede tener inestabilidad hemodinámica o disfunción múltiple de órganos relacionados con pancreatitis grave. El paciente puede presentar dolor abdominal, signos de exsanguinante pérdida de sangre, o sangrado intermitente lento. Un proceso exanguinante súbita, lo que conduce a la muerte en cuestión de minutos a horas, se ha informado en 7,5\% de los pacientes con rupturas seudoaneurisma. (Flati G A.-S. A., 2003)

\section{Trombosis venosa}

La trombosis venosa se puede observar en la pancreatitis aguda y se relaciona con procesos inflamatorios agudos del páncreas adyacente a las estructuras venosas. El aislado de trombosis de la vena esplénica es el tipo más común, y, en algunas series de casos, ha tenido una incidencia tan alta como $42 \%$ en los casos de pancreatitis aguda. (Mauro MA, 2013). Menos comúnmente, portal y trombosis de la vena mesentérica superior puede ocurrir como resultado de pancreatitis. La patogénesis está relacionada con una lesión de la íntima y la compresión como consecuencia de la proximidad de la vasculatura venosa al páncreas, específicamente la vena 


\section{Complicaciones más frecuentes en pacientes con pancreatitis}

Vol. 3, núm. 3., (2019)

Lissette Esther Villagrán Rodríguez; Raúl Enrique Carrión Sánchez; Aliz Janeth Calero Correa; Dolores Mariela Perero Briones

esplénica. El estasis de flujo de la sangre se produce a partir de estos factores intrínsecos y extrínsecos y, finalmente, conduce a la trombosis. En el desarrollo de estasis venosa se ha planteado la hipótesis de que se produzca como resultado de la compresión por pseudoquistes y / o parénquima de páncreas edematoso ampliada. La obstrucción de la vena esplénica puede ser vista en la cola pseudoquiste en casi el 30\% de los casos. (Bernades P, 2012)

\section{Otras complicaciones vinculadas con sangrado}

Otras complicaciones hemorrágicas incluyen sangrado posnecrosectomía, que puede ser el resultado de desbridamiento demasiado agresivo y la colocación y / o el uso de tubos de drenaje que no cumplen al lado de una estructura vascular que resulta en necrosis por presión y la erosión directa en un recipiente. En algunos casos, el desbridamiento incompleto puede resultar en la sepsis y la erosión en las estructuras vasculares, así, datos sugieren que las complicaciones hemorrágicas son significativamente más común en las intervenciones que se producen en la fase aguda versus intervenciones después de retraso. (Kriwanek S, 2011). La arterioembolizacion radiológica es la principal estancia de gestión reservando en la cirugía para los casos refractarios o para pacientes con inestabilidad hemodinámica. En raras ocasiones, el sangrado puede ocurrir en el conducto pancreático, lo que se conoce como pancreaticus hemosuccus; sin embargo, esto se ve más típicamente como una complicación de la pancreatitis crónica gastrointestinal intermitente, de bajo grado de sangrado hasta sangrado exsanguinante, lo cual ha sido descrito como un resultado de pancreaticus hemosuccus. Debido a la rara ocurrencia, hay datos limitados sobre su gestión. Las opciones incluyen arterioembolizacion radiográfica frente quirúrgico recipiente de ligadura de sutura con o sin pancreatectomía distal. Las tasas de mortalidad asociadas con la cirugía se han reportado tan alto como $20 \% 25 \%$, a 


\section{Complicaciones más frecuentes en pacientes con pancreatitis}

Vol. 3, núm. 3., (2019)

Lissette Esther Villagrán Rodríguez; Raúl Enrique Carrión Sánchez; Aliz Janeth Calero Correa;

Dolores Mariela Perero Briones

pesar de resangrado las tasas son significativamente más baja que la de arterioembolización. (Sukanta Ray, 2011)

\section{Otras complicaciones de la pancreatitis}

Las complicaciones esplénicas aisladas de la pancreatitis aguda son infrecuentes. pseudoquistes intraesplénicos, esplénica de miocardio, la necrosis, ruptura y hematoma han sido reportados como posibles complicaciones. Algunas de estas complicaciones pueden ser potencialmente mortales y requieren esplenectomía emergente, y si es necesario, la pancreatectomía distal como tratamiento definitivo. (Sukanta Ray, 2011)

La compresión del intestino ha sido reportada como un resultado de la necrosis por presión de los desechos inflamatorios de la cola del páncreas. Esto puede resultar en signos y síntomas de obstrucción parcial o completa y puede incluso resultar en fistulización del intestino delgado o grueso. El colon izquierdo es el sitio más común de compresión y fistulización. (Alridge MC, 2007). La descompresión de la lesión obstructiva se puede hacer por vía percutánea, endoscópica o quirúrgicamente dependiendo del tipo de lesión. Después de haberse producido fistulización, el tratamiento se limita a la reparación o resección quirúrgica. La obstrucción del conducto biliar común puede ser una complicación de la pancreatitis aguda, esto se ve normalmente en la fase sintomática temprana de la enfermedad y se relaciona con la masa inflamatoria en la cabeza del páncreas, pseudoquistes, o colecciones de líquido. Típicamente, el conducto biliar común distal se ve afectado y se calma con la mejoría clínica de la morbilidad y se debe considerar la terapia de primera línea. Los pacientes generalmente necesitan de descompresión a corto plazo con stents o catéteres como en la resolución, se ve casi siempre 


\section{Complicaciones más frecuentes en pacientes con pancreatitis}

Vol. 3, núm. 3., (2019)

Lissette Esther Villagrán Rodríguez; Raúl Enrique Carrión Sánchez; Aliz Janeth Calero Correa; Dolores Mariela Perero Briones

mejoría una vez que se produce el cuadro clínico de la enfermedad subyacente. En los pacientes que desarrollan sepsis biliar o prurito intratable, las opciones de gestión incluyen endoscópicos, quirúrgicos o descompresión percutánea. La endoscópica o descompresión percutánea se asocia con una menor morbilidad y se debe considerar la terapia de primera línea. Los pacientes generalmente necesitan de descompresión a corto plazo con stents o catéteres como la resolución se ve casi siempre mejora una vez que se produce clínico de la enfermedad subyacente.

\section{Conclusiones.}

En conclusión, la pancreatitis aguda es un proceso inflamatorio agresivo que puede tener afectación de órganos regional y a distancia variable. Las complicaciones locales de la pancreatitis son muchos y pueden resultar en mayor o menor grado de morbilidad y mortalidad o en la aparición de las complicaciones que pueden ocurrir en cualquier momento durante el curso de la enfermedad aguda. La supervisión cuidadosa de los pacientes tomadas para reconocer rápidamente las complicaciones en última instancia, puede cambiar el curso de la enfermedad. El manejo de las complicaciones es guiado por la identificación y la comprensión de las complicaciones específicas y también en el conocimiento y la disponibilidad de los especialistas que tratan a pacientes con esta enfermedad.

\section{Bibliografía.}

Adams DB, A. M. (2015). Drenaje del catéter en comparación con el drenaje inter-nal en la gestión de seudoquistes pancreáticos. . Chile: Ann Surg.

Alridge MC, F. N. (2007). Complicaciones colónica de pancreatitis aguda grave. . 62-69.

Arvanitakis M, D. M. (2007). Deviere tratamiento J de fístulas pancreáticas externos: cuando el drenaje del conducto pancreático principal no es enough. EE.UU: Am J Gastroentero. 


\section{Complicaciones más frecuentes en pacientes con pancreatitis}

Vol. 3, núm. 3., (2019)

Lissette Esther Villagrán Rodríguez; Raúl Enrique Carrión Sánchez; Aliz Janeth Calero Correa;

Dolores Mariela Perero Briones

Baron TH, H. G. (2002). Diferencias en resultado después de drenaje endoscópico de la necrosis pancreática, seudoquistes pancreáticos agudas y crónicas seudoquistes pancreáticos. Mexico: Gastrointest Endos.

Berger HG, R. B. (2003). Historia natural de la necrotizante pan-creatitis. Pancreatology . Buenos Aires.

Bernades P, B. A. (2012). Esplénica y obstrucción venosa portal en la pancreatitis crónica. Un estudio longitudinal prospectivo de una serie médico-quirúrgico de 266 pacientes. Mexico: Dig Dis Sci .

Bolívar, J. (2015). Investigación Documental. México. Pax.

Bradley L, C. J. (2011). La historia natural de los seudoquistes pancreáticos: un concepto unificado de gestión. los seudoquistes pancreáticos: un concepto unificado de gestión. Lima: Am J Surg.

Brooks, R. (2013). Psuedocysts del páncreas. En: Cirugía del pancreas. Philadelphia: JR brooks.

Buchler MW, G. B. (2000). Estrategia de tratamiento de acuerdo con el estado de la infección: necrotizante pancreatitis aguda. . Guatemala: Ann Surg.

Cameron, J. (2014). Pancreatitis aguda. En: Cirugía del Tracto Alimentario, Shackelford, RT, Zuideme, GD (Eds), WB Saunders. Philadelphia: $2^{\text {a }}$ edición.

Castro, J. (2016). Técnicas Documentales. México. Limusa.

Catalano M, G. J. (2015). Tratamiento de seudoquistes pancreáticos con la comunicación ductal por transpapilar endoprótesis conducto pancreático. . Gastrointest Endosc , 42-114.

Cervantes A, G. F. (2004). Drenaje laparoscópico de seudoquistes pancreáticos. Surg Endosc.

Davila, A. (2015). Diccionario de Términos Científicos. . Caracas: Editorial Oasis.

Dervenis C, J. C. (2012). Diagnóstico evaluación objetiva de la gravedad y el tratamiento de la pancreatitis aguda. Mexico: Int J Pancreatol.

Flati G, A.-S. A. (2003). Hemorragia potencialmente mortal en la pancreatitis aguda: fisiopatología, prevención, y tratamiento. Páncreas . 8-14.

Flati G, S. F. (2015). Complicaciones hemorrágicas graves en la pancreatitis. 45-89.

Kriwanek S, G. M. (2011). Las complicaciones después de la cirugía para la pancreatitis necrotizante: factores de riesgo y el pronóstico. . Mexico: Eur J Surg. 


\section{Complicaciones más frecuentes en pacientes con pancreatitis}

Vol. 3, núm. 3., (2019)

Lissette Esther Villagrán Rodríguez; Raúl Enrique Carrión Sánchez; Aliz Janeth Calero Correa; Dolores Mariela Perero Briones

Lawson M, B. J. (2011). : El tratamiento endoscópico para pseudo-quistes pancreáticos. . EE.UU: Gastrointest Endosc Clin North.

Mauro MA, S. M. (2013). El bazo y su vasculatura en la pancreatitis: hallazgos de la TC. 15-27.

Medicine, U. (13 de Marzo de 2018 ). El páncreas: Anatomía y funciones. Recuperado el 3 de Julio de 2019, de http://healthlibrary.uchospitals.edu/content/adult-diseases-andconditions-v0/pand225ncreas-anatomand237a-y-funciones/

Parque E, H. B. (2002). Laparoscopia terapéutica del páncreas. 2002; 236: 149. Ann Surg , 3649.

Sukanta Ray, K. D. (2011). Hemouccus pancreaticus Asociado con pancreatitis aguda severa y pseudoaneurismas: Un reporte de dos casos. . 69-78.

Tercero, B. (2013). Un sistema de clasificación basado clínicamente para la pancreatitis aguda. Bogota: Arco Surg.

Vitas GJ, S. M. (2002). Tratamiento quirúrgico versus tratamiento expectante: la gestión de los seudoquistes pancreáticos seleccionado. . Bogota: Cirugía.

Yeo J, B. J.-N. (2010). La historia natural de seudoquistes pancreáticos documentados por tomografía computarizada. 15-39.

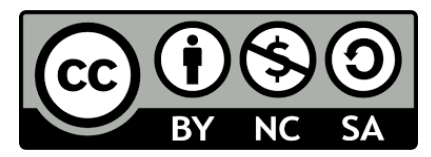

RECONOCIMIENTO-NOCOMERCIAL-COMPARTIRIGUAL

CC BY-NC-SA

ESTA LICENCIA PERMITE A OTROS ENTREMEZCLAR, AJUSTAR Y CONSTRUIR A PARTIR DE SU OBRA CON FINES NO COMERCIALES, SIEMPRE Y CUANDO LE RECONOZCAN LA AUTORÍA Y SUS NUEVAS CREACIONES ESTÉN BAJO UNA LICENCIA CON LOS MISMOS TÉRMINOS. 